\title{
TRANSIENT MARKOV CHAINS WITH STATIONARY MEASURES
}

\author{
T. E. HARRIS
}

1. Introduction and summary. We consider Markov chains $x_{n}$, $n=0,1, \cdots$, with denumerable states denoted by integers $0,1, \cdots$. Let $P_{i j}=P_{i j}^{1}=\operatorname{Pr}\left\{x_{n+1}=j \mid x_{n}=i\right\}$ and let $P_{i j}^{n}$ denote the $n$-step transition probability from $i$ to $j, n=1,2, \cdots$. It is assumed throughout that for each $i, j$ there is an $n$ such that $P_{i j}^{n}>0$. For other terminology see Feller [5, Chapter 15]. (We shall refer to chains satisfying the above assumption as irreducible.)

We shall be concerned with the existence of solutions of the "equations of stationarity,"

$$
Q_{i}=\sum_{j=0}^{\infty} Q_{j} P_{j i}, \quad i=0,1, \cdots,
$$

in the case where the chain is transient. A solution will always mean a set of positive numbers satisfying (1.1). (It is readily seen that if a set of non-negative numbers, not all 0 , satisfy (1.1) they must all be strictly positive.) If the chain has finite mean recurrence times it is known that there is a solution whose elements $Q_{i}$ are a set of probabilities, $\sum Q_{i}=1$. See [5]. If it is assumed only that the chain is recurrent, Derman showed, [3], that there is a unique (up to a constant multiplier) solution, with $\sum Q_{i}=\infty$ in case the mean recurrence times are infinite. Derman also showed by examples in [4] that if the chain is transient there may or may not be solutions.

In this note we obtain a necessary condition and a sufficient condition for the existence of a solution of (1.1). The author thinks that the sufficient condition is in some sense close to being necessary. The main results are in Theorems 1 and 2. The corollary to Theorem 2 covers a number of cases of interest. This corollary also follows from general results, in a somewhat different direction, of Edward Nelson. (Private communication.)

2. A necessary condition. We shall use the following terminology:

Definition 1. A path from infinity is a sequence of states $i_{1}, i_{2}, \cdots$, not necessarily all distinct but containing infinitely many distinct states, such that

$$
\operatorname{Pr}\left\{x_{n+1}=i_{k} \mid x_{n}=i_{k+1}\right\}>0, \quad k=1,2, \cdots .
$$

Received by the editors December 19, 1955. 
Definition 2. A simple path from infinity is a path from infinity, all of whose states are distinct.

THEOREM 1. In order that (1.1) should have a solution for an irreducible transient chain, it is necessary that there exist a simple path from infinity.

Proof. Suppose (1.1) has a solution $\left\{Q_{i}\right\}$. Define a set of "inverse probabilities" $p_{i j}$ by

$$
p_{i j}=P_{j i} Q_{j} / Q_{i}, \quad p_{i j}^{n}=P_{j i}^{n} Q_{j} / Q_{i} .
$$

Since $\sum_{n} p_{i j}^{n}=\left(Q_{j} / Q_{i}\right) \sum_{n} P_{j i}^{n}<\infty$, the chain defined by the $p_{i j}$ is transient. Let $y_{n}, n \geqq 0$, be the variables of such a chain. It is evident that for almost every sample sequence $y_{0}, y_{1}, \cdots$, the quantities $p_{y_{0} y_{1}}, p_{y_{1} y_{2}} \cdots$ are all positive. Moreover, because of the transient character of the chain, it is true for almost every sequence that no state is visited infinitely often. Therefore, almost every sequence contains a subsequence $y_{0}^{\prime}, y_{1}^{\prime}, \cdots$, such that the $y_{i}^{\prime}$ are all distinct and $p_{y_{i}^{\prime} v_{i+1}^{\prime}}>0$. Referring to (2.1) we see that there is thus a simple path from infinity. This completes the proof of Theorem 1.

As an example, consider the renewal process, for which Derman showed directly that there is no solution in the transient case. Here $p_{i j}=0$ unless $j=0$ or $i+1$, and it is evident that there is no simple path from infinity. In the renewal process the state 0 has a special role; every path from infinity must contain it infinitely of ten. However, it can be shown by examples that even if there is no simple path from infinity, there need not exist any such distinguished state. In fact, an example, which we do not give here, shows that even if no finite set of states has the property that every path from infinity intersects it infinitely often, there need exist no simple path from infinity.

3. Conditions on the zeros. The condition of Theorem 1 is not sufficient for the existence of a solution to (1.1). Note that this is a condition on the location of the zeros in the matrix $\left(P_{i j}\right)$. The following two examples show that no condition on the zeros can be both necessary and sufficient for the existence of a solution in the transient case, since the zeros of the two examples are in the same place and one has a solution while the other does not.

EXAMPLE 1. Take $P_{i, i+1}=1-e^{-i}-e^{-i^{8}}, \quad P_{i, i-1}=e^{-i}, \quad P_{i 0}=e^{-i^{3}}$, $i=1,2, \cdots ; P_{01}=1$. This chain is clearly transient and irreducible. Theorem 2 below can be applied to show that there is a solution to (1.1). We shall not give the details. 
EXAmple 2. Take $P_{i, i+1}=1-(1 / 2)^{i}, P_{i, i-1}=(1 / 4)^{i}, P_{i 0}=(1 / 2)^{i}$ $-(1 / 4)^{i}, i=1,2, \cdots ; P_{01}=1$. This chain is likewise transient and irreducible. We now prove that in this case (1.1) has no solution.

Proof that THERE IS No SOlution for Example 2. Suppose that a solution $\left\{Q_{i}\right\}$ exists. First, the $Q_{i}$ cannot be bounded. For, setting $i=0$ in (1.1), we have

$$
\begin{aligned}
Q_{0} & =\sum_{j=0}^{\infty} Q_{j} P_{j 0}=\sum_{j=0}^{\infty} Q_{j} P_{j 0}^{n}, & \\
N Q_{0} & =\sum_{j=0}^{\infty} \sum_{n=1}^{N} Q_{j} P_{j 0}^{n}, & N=1,2, \cdots .
\end{aligned}
$$

If the $Q_{j}$ were bounded by $K$ we would have

$$
N Q_{0} \leqq K \sum_{j=0}^{\infty} \sum_{n=1}^{N} P_{j 0}^{n}<K \sum_{j=0}^{\infty} \sum_{n=1}^{\infty} P_{j 0}^{n} .
$$

Now $\sum_{n=1}^{\infty} P_{j 0}^{n}$ is the expected total number of visits to state 0 of a particle starting in state $j$ and is equal to $L_{j 0}$ multiplied by the expected number of visits to 0 starting in 0 , where $L_{j 0}$ is the probability of reaching 0 from $j$ at least once. It is readily seen that $\sum_{j} L_{j 0}<\infty$, which means that (3.2) cannot hold for all $N$. Hence the $Q_{i}$, if they exist, must be unbounded. Next, observe that equations (1.1) have the form in this example

$$
\begin{aligned}
& Q_{0}=\sum_{j=0}^{\infty}\left[\left(\frac{1}{2}\right)^{i}-\left(\frac{1}{4}\right)^{i}\right] Q_{j}, \quad Q_{1}=Q_{0}+\left(\frac{1}{4}\right)^{2} Q_{2} \\
& Q_{i}=\left[1-\left(\frac{1}{2}\right)^{i-1}\right] Q_{i-1}+\left(\frac{1}{4}\right)^{i+1} Q_{i+1}, \quad i=2,3, \cdots
\end{aligned}
$$

Since $1-(1 / 2)^{i-1}+(1 / 4)^{i+1}<1$ for $i \geqq 2$, we see from (3.4) that $Q_{i}$ cannot be as large as $\operatorname{Max}\left(Q_{i-1}, Q_{i+1}\right), i=2,3, \cdots$. Since the $Q_{i}$ are unbounded they must therefore ultimately increase monotonically to infinity. Hence, there is an $i$ such that if $j \geqq i, Q_{j} \geqq Q_{j-1}$. Then, from (3.4)

$$
\begin{gathered}
Q_{j+1}=\frac{Q_{j}-\left[1-\left(\frac{1}{2}\right)^{j-1}\right] Q_{j-1}}{(1 / 4)^{j+1}} \geqq \frac{\left(\frac{1}{2}\right)^{j-1} Q_{j-1}}{(1 / 4)^{j+1}} \\
=2^{j+3} Q_{j-1}, \quad j=i, i+1, \cdots .
\end{gathered}
$$

By repeated application of (3.5) we have, after a little simplification, $Q_{i+2 n+1}>2^{n^{2}} Q_{i-1}, n=1,2, \cdots$. This implies that the sum in (3.3) is 
infinite, a contradiction. Hence (1.1) can have no solution.

\section{A sufficient condition.}

Definitions. Let ${ }_{k} P_{i j}^{1}=P_{i j}$, and in general

(4.1) ${ }_{k} P_{i j}^{n}=\operatorname{Pr}\left\{x_{n}=j ; x_{m} \neq k, 1 \leqq m<n \mid x_{0}=i\right\}, n=1,2, \cdots$; and define

$$
L_{k i}(j)=\sum_{r=j}^{\infty} \sum_{n=1}^{\infty}{ }_{i} P_{k r}^{n} P_{r i}+P_{k i} .
$$

$L_{k i}(j)$ is, if $k \geqq j$, the probability that a particle, starting at $k$, will reach $i$, the first visit being immediately preceded by a state with index $\geqq j$. As before, $L_{k i}=L_{k i}(0)$ will denote the probability of reaching $i$ at all, having started from $k$.

THEOREM 2. In order that (1.1) should have a solution for an irreducible transient chain, the following condition is sufficient: there exists an infinite set $K$ of states such that

$$
\lim _{j \rightarrow \infty, \boldsymbol{k} \rightarrow \infty, k \in K} L_{k i}(j) / L_{k i}=0, \quad i=0,1,2, \cdots .
$$

COROLlARY. If the chain is transient and irreducible, and if for each $i$, $P_{k i}=0$ except for a finite set of values of $k$, then (1.1) has a solution.

Proof of Theorem 2. In the recurrent case Chung [2] showed that we can pick an arbitrary state, say 0 , and define $Q_{i}^{\prime}=\sum_{n=1}^{\infty} P_{0 i}^{n}$, obtaining a solution. Here $Q_{i}^{\prime}$ is the expected number of visits to $i$ between visits to 0 , and we have (note that because of recurrence $\left.Q_{0}^{\prime}=1\right)$

$$
\begin{aligned}
\sum_{n=1}^{\infty}{ }_{0} P_{0 i}^{n} & ={ }_{0} P_{0 i}^{1}+\sum_{n=2}^{\infty} \sum_{j=1}^{\infty}{ }_{0} P_{0 j}^{n-1} P_{j i} \\
& ={ }_{0} P_{0 i}^{1}+\sum_{j=1}^{\infty} Q_{j}^{\prime} P_{j i}=\sum_{j=0}^{\infty} Q_{j}^{\prime} P_{j i}
\end{aligned}
$$

since ${ }_{0} P_{0 i}^{1}=P_{0 i}^{1}=Q_{0}^{\prime} P_{0 i}^{1}$. In the transient case this does not give a solution. It seems reasonable to try instead

$$
Q_{i}^{\prime \prime}=\sum_{n=1}^{\infty} P_{k i}^{n}
$$

the expected total number of visits to $i$, starting at some state $k$. It is perhaps better to think of $Q_{i}^{\prime \prime}$ as the average density of particles at $i$, if there is a source putting one particle per time unit into the sys- 
tem at $k$. Although $Q_{i}^{\prime \prime}$ is not itself a solution, we may hope that it approaches a solution as the "source" $k$ moves off to infinity. We must also normalize to keep the $Q_{i}$ within bounds. Hence we define

$$
Q_{k i}=\sum_{n=1}^{\infty} P_{k i}^{n}, \quad i, k=0,1, \cdots .
$$

We shall show that if the conditions of Theorem 2 hold, then there is a sequence $\left\{k_{m}\right\}$ such that

$$
\lim _{m \rightarrow \infty} Q_{k_{m}, i} / Q_{k_{m}, 0}=Q_{i} \quad i=0,1, \cdots,
$$

where the $Q_{i}$ are a solution of (1.1).

Definitions. Let $\theta_{i j}, i \neq j$, be the probability that the state, given to be initially $i$, reaches $j$, before reaching $i$; let $1-\phi_{i j}, i \neq j$, be the probability that the state, initially $i$, returns to $i$ without reaching $j$.

In general, $\theta_{i j} \neq \phi_{i j}$ for transient chains. Clearly the $\theta_{i j}$ and the $\phi_{i j}$ are all positive.

Now suppose the state is initially $i \neq j$, and let $V$ be the total number of visits to $j$ which precede any further visit to $i$. Then clearly

$$
E(V)=\theta_{i j} / \phi_{j i} \text {. }
$$

Hence we have

$$
Q_{k j}=Q_{k i} \theta_{i j} / \phi_{j i}+\sum_{n=1}^{\infty}{ }_{i} P_{k j}^{n}
$$

Then

$$
\begin{aligned}
Q_{k i}= & \sum_{n=1}^{\infty} P_{k i}^{n}=P_{k i}+\sum_{n=2}^{\infty} \sum_{r=0}^{\infty} P_{k r}^{n-1} P_{r i}=P_{k i}+\sum_{r=0}^{j-1} Q_{k r} P_{r i} \\
& +\sum_{r=j}^{\infty}\left[Q_{k i} \frac{\theta_{i r}}{\phi_{r i}}+\sum_{n=1}^{\infty}{ }_{i} P_{k r}^{n}\right] P_{r i},
\end{aligned}
$$

Dividing both sides of (4.9) by $Q_{k 0}$, and recalling (4.2), we obtain

$$
\frac{Q_{k i}}{Q_{k 0}}=\sum_{r=0}^{i-1}\left(\frac{Q_{k r}}{Q_{k 0}}\right) P_{r i}+\frac{Q_{k i}}{Q_{k 0}} \sum_{r=j}^{\infty}\left(\frac{\theta_{i r}}{\phi_{r i}}\right) P_{r i}+\frac{L_{k i}(j)}{Q_{k 0}}, \quad j>i .
$$

From (4.8) we see that $Q_{k j} / Q_{k i}>\theta_{i j} / \phi_{j i}$; interchanging $i$ and $j$ gives

$$
\theta_{i j} / \phi_{j i}<Q_{k j} / Q_{k i}<\phi_{i j} / \theta_{j i}
$$

Hence ratios such as $Q_{k r} / Q_{k 0}$ are bounded away from 0 and $\infty$ by numbers which may depend on $r$ but are independent of $k$. Also, 
$Q_{k i}=L_{k i}\left(1+Q_{i i}\right)$. Thus, the condition of Theorem 2 implies that if $k \in K, k \rightarrow \infty, j \rightarrow \infty$, then $L_{k i}(j) / Q_{k 0} \rightarrow 0$ for each $i$. The above remarks also imply that there is a sequence $\left\{k_{m}\right\}$ independent of $i, m=1,2, \cdots$, with $k_{m} \in K, k_{m} \rightarrow \infty$, such that

$$
\lim _{m \rightarrow \infty} \frac{Q_{k_{m}, i}}{Q_{k_{m}, 0}}=Q_{i}, \text { say, }
$$

exists for each $i$. Since for each $i$ the ratio $Q_{k i} / Q_{k 0}$ is a bounded function of $k$, and since, referring to (4.10),

$$
\sum_{r=j}^{\infty}\left(\theta_{i r} / \phi_{r i}\right) P_{r i}
$$

is arbitrarily small for $j$ sufficiently large, the limits $Q_{i}$ must satisfy (1.1). This concludes the proof of Theorem 2.

5. Remarks. Blackwell [1] was concerned with the number of bounded solutions for transient chains of the system

$$
q_{i}=\sum_{j=0}^{\infty} P_{i j} q_{j}
$$

The relation between solutions of (5.1) and of (1.1) is discussed by Feller [6].

\section{REFERENCES}

1. D. Blackwell, On transient Markov processes with a countable number of states and stationary transition probabilities, Ann. Math. Statist. vol. 26 (1955) pp. 654-658.

2. K. L. Chung, Contributions to the theory of Markov chains II, Trans. Amer. Math. Soc. vol. 76 (1954) pp. 397-419.

3. C. Derman, $A$ solution to a set of fundamental equations in Markov chains, Proc. Amer. Math. Soc. vol. 5 (1954) pp. 332-334.

4. - Some contributions to the theory of denumerable Markov chains, Trans. Amer. Math. Soc. vol. 79 (1955) pp. 541-555.

5. W. Feller, An introduction to probability theory and its applications, New York, Wiley, 1950.

6. W. Feller, Boundaries induced by stochastic matrices, Trans. Amer. Math. Soc. vol. 83 (1956) pp. 19-54.

RAND CORPORATION 